\title{
The Drell-Yan measurement at COMPASS-II
}

\section{Stefano Takekawa*}

Istituto Nazionale di Fisica Nucleare

E-mail: takekawa@to.infn.it

The Drell-Yan process can be used to access Transverse Momentum Dependent Parton Distribution Functions (TMD PDFs), such as the Sivers and the Boer-Mulders functions, as well as the transversity function providing complementary information to what is known from Semi Inclusive Deep Inelastic Scattering (SIDIS) data. The COMPASS experiment offers the possibility to study single polarized Drell-Yan processes $\left(\pi^{-}+\mathrm{p}^{\uparrow} \rightarrow \mu \mu+\mathrm{X}\right)$, making use of its large acceptance spectrometer and its unique transversely polarised proton target. Moreover a fundamental test of the factorization theorem in the non-perturbative QCD can be performed, by verifying the sign change of the T-odd Boer-Mulders and Sivers functions depending if they are accessed via SIDIS or Drell-Yan process. As the start of the Drell-Yan program at COMPASS is approaching, foreseen in late 2014, the spectrometer has been updated to fulfill the needs of this measurement

Photon 2013,

20-24 May 2013

Paris, France

${ }^{*}$ Speaker. 


\section{Introduction}

The spin structure of the nucleon has been investigated by the COMPASS Collaboration by studying Semi Inclusive Deep Inelastic Scattering (SIDIS) of muons on longitudinally and transversely polarised nucleons. The new COMPASS-II Proposal [1] extends the COMPASS physics program by exploring two new processes to investigate the nucleon spin structure: Deeply Virtual Compton Scattering (DVCS) and single polarised Drell-Yan. After the approval of the CERN Research Board on 1st December 2010, a preliminary schedule foresees the start of the Drell-Yan program at COMPASS in late 2014.

\section{The Drell-Yan process and TMDs}

At leading order the proton can be described by eight Transverse Momentum Dependent Parton Distribution Functions (TMD PDFs), when one permits non-zero intrinsic transverse momentum $\mathbf{k}_{T}$ of partons inside the nucleons. They are: $f_{1}\left(x, \mathbf{k}_{T}^{2}\right), g_{1 L}\left(x, \mathbf{k}_{T}^{2}\right), h_{1}\left(x, \mathbf{k}_{T}^{2}\right), g_{1 T}\left(x, \mathbf{k}_{T}^{2}\right)$, $h_{1 L}^{\perp}\left(x, \mathbf{k}_{T}^{2}\right), h_{1 T}^{\perp}\left(x, \mathbf{k}_{T}^{2}\right), h_{1}^{\perp}\left(x, \mathbf{k}_{T}^{2}\right)$ and $f_{1 T}^{\perp}\left(x, \mathbf{k}_{T}^{2}\right)$, where $x$ is the fraction of the proton momentum carried by the partons. The TMD PDFs describe the correlation of momentum and spin of partons with the spin of the parent proton if it is either unpolarised or longitudinally/transversely polarised, so their study can help to understand the helicity structure of nucleon and to solve the long lasting spin puzzle. The first three functions listed above, when integrated over $\mathbf{k}_{T}$, yields to the momentum distribution, the helicity distribution and transversity function, commonly known in the collinear approximation. The other five TMD PDFs, as well as the transversity function, are chiral-odd. In order to measure them, one needs to observe processes whose cross sections contain them convoluted with other chiral-odd quantities to get observables. The TMD PDFs, like the Pretzelosity $h_{1 T}^{\perp}\left(x, \mathbf{k}_{T}^{2}\right)$, the Boer-Mulders $h_{1}^{\perp}\left(x, \mathbf{k}_{T}^{2}\right)$ and the Sivers $f_{1 T}^{\perp}\left(x, \mathbf{k}_{T}^{2}\right)$ functions, can be accessed studying the Semi Inclusive Deep Inelastic (SIDIS) scattering, whose cross section can be expressed as the sum of convolution of TMD PDFs and fragmentation functions. The cross section of the Drell-Yan process can be expressed by the sum of convolutions of only TMD PDFs. Therefore the SIDIS and the Drell-Yan processes give complementary ways to probe the structure of the proton. The Boer-Mulders and the Sivers functions are naïvely T-odd; as a consequence, QCD factorization predicts that both functions should change their sign when measured in SIDIS experiments and in Drell-Yan experiments [2], thus an important test of QCD can be performed, since the relations should hold:

$$
\begin{aligned}
f_{1 T}^{\perp}(D Y) & =-f_{1 T}^{\perp}(\text { SIDIS }) \\
h_{1}^{\perp}(D Y) & =-h_{1}^{\perp}(\text { SIDIS })
\end{aligned}
$$

The Drell-Yan process is the annihilation of a quark-antiquark pair coming from two hadrons into a virtual photon which produces a pair of leptons [3]. The angular distribution of the lepton was widely studied in the past. One of the results is the so-called Lam-Tung sum rule [4], which says $1-\lambda=2 v . \lambda, \mu$ and $v$ are three parameters which appears in the expression of the unpolarised angular distribution of the leptons:

$$
\frac{1}{\sigma} \frac{d \sigma}{d \Omega}=\frac{3}{4 \pi} \frac{1}{\lambda+3}\left(1+\lambda \cos ^{2} \theta+\mu \sin ^{2} \theta \cos \phi+\frac{v}{2} \sin ^{2} \theta \cos 2 \phi\right)
$$




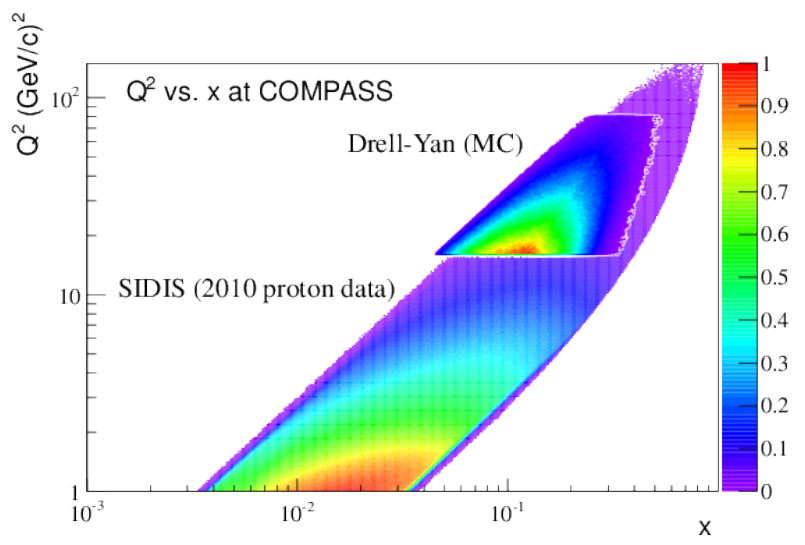

Figure 1: The face spaces of the SIDIS and Drell-Yan processes overlap at COMPASS. The extraction of TMD PDFs is done in the same $Q^{2}$ and $x$ region.

In the collinear approximation, the QED predicts $\lambda=1$ and $\mu=v=0$. However measurements are not in agreement with the QED prediction [5] [6], with non-zero values for $\mu$ and $v$, and even the validity of the sum rule was put in doubt. The distributions of azimuthal angles between the lepton and hadron planes, as well as between the lepton plane and the spin of the polarised hadron, provide information on the TMD PDFs in the Drell-Yan process [7]. In case of the reaction $\pi^{-}+p^{\uparrow} \rightarrow \mu^{+} \mu^{-}+X$ (single polarised Drell-Yan), with a pion beam scattering on a (polarised) proton, four asymmetry terms can be isolated to access TMDs of the two hadrons in the angular distribution is [1]:

$$
\begin{aligned}
\frac{\mathrm{d} \sigma}{\mathrm{d}^{4} q \mathrm{~d} \Omega} & =\frac{\alpha_{e m}^{2}}{F q^{2}} \hat{\sigma}_{U}\left\{\left(1+D_{\left[\sin ^{2} \theta\right]} A_{U}^{\cos 2 \phi} \cos 2 \phi\right)\right. \\
& +\left|\mathbf{S}_{T}\right|\left[A_{T}^{\sin \phi_{S}} \sin \phi_{S}+D_{\left[\sin ^{2} \theta\right]}\left(A_{T}^{\sin \left(2 \phi+\phi_{S}\right)} \sin \left(2 \phi+\phi_{S}\right)\right.\right. \\
& \left.\left.\left.+\quad A_{T}^{\sin \left(2 \phi-\phi_{S}\right)} \sin \left(2 \phi-\phi_{S}\right)\right)\right]\right\}
\end{aligned}
$$

where $D_{[f(\theta)]}$ are the depolarisation factors, $\mathbf{S}_{T}$ is the target polarisation vector, $F$ is the flux of incoming hadrons, the $A \mathrm{~s}$ are the azimuthal asymmetries, $\hat{\sigma}_{U}$ is the part of the cross section which survives the integration over $\phi$ and $\phi_{S}, \phi$ and $\theta$ are the azimuthal and polar angles of the lepton in the Collins-Soper frame [8] and $\phi_{S}$ is the angle of the spin in the proton rest frame. Each asymmetry term gives access to the convolution of two TMD PDFs: in $A_{U}^{\cos 2 \phi}$ of the Boer-Mulders functions of the incoming hadrons, in $A_{T}^{\sin \phi_{S}}$ of the Sivers function of the target nucleon and unpolarised of beam particle, in $A_{T}^{\sin \left(2 \phi+\phi_{S}\right)}$ of the Boer-Mulders function of the beam hadron and to the Pretzelosity function of the target nucleon, in $A_{T}^{\sin \left(2 \phi-\phi_{S}\right)}$ of the Boer-Mulders function of the beam hadron and the transversity function of the target nucleon.

\section{The COMPASS spectrometer}

COMPASS is a fixed target experiment located in the North Area at CERN [9] [10]. The COMPASS spectrometer is built at the end of the M2 beam line which can deliver a hadron beam to the polarised target. The spectrometer has a large acceptance, up to $\pm 180 \mathrm{mrad}$ thanks to its 
two stages structure. Each stage is built around an analysing magnet and it is equiped with tracking detectors (GEM, MicroMegas, MWPC, Straws, SciFi and Silicon detectors) and hadronic and electromagnetic calorimeters. The first stage has also a RICH detector for particle identification while muon filters provide muon identification in both stages. The unique polarised target is capable to polarise ammonia (proton target) up to $90 \%$ with a dilution factor of 0.22 (for the Drell-Yan process). The COMPASS spectrometer gives the possibility to study the Drell-Yan process in the reaction $\pi^{-}+p^{\uparrow} \rightarrow \mu^{+} \mu^{-}+X$.

\section{The spectrometer upgrades}

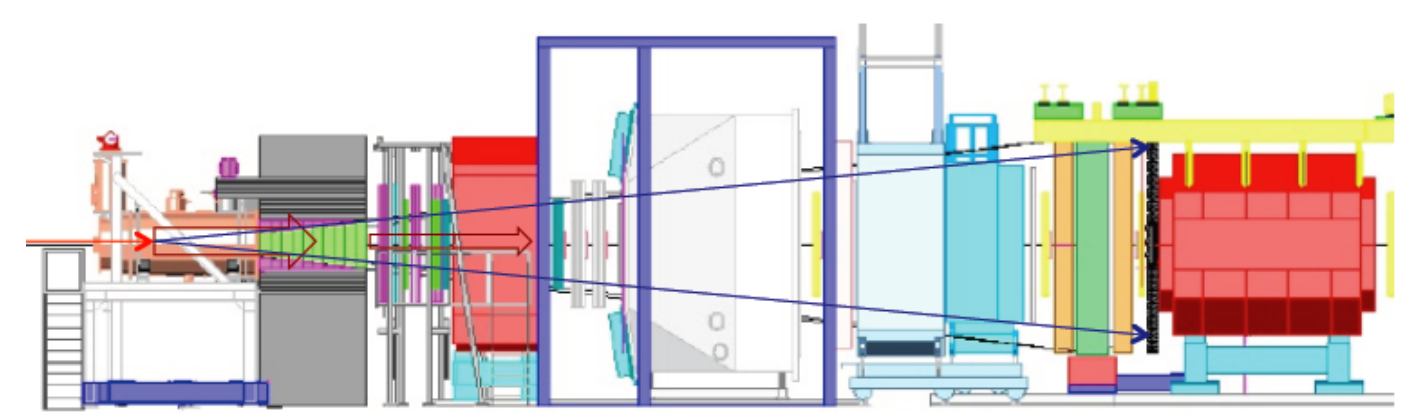

Figure 2: Side view of the first stage of the COMPASS spectrometer for the Drell-Yan measurement. The beam comes from the left and scatter of the target. In the picture from left to right: target (orange), absorber (violet and grey), detectors, SM1 magnet (red), RICH detector (grey inside blue structure), calorimeters, muon filter, SM2 magnet (red).

Minor upgrades of the COMPASS spectrometer are required to make it efficient for the future Drell-Yan experiment. The most important one is the installation of a hadron absorber after the target, to significantly reduce the secondary particle flux and to suppress the background from the pion decay. In fact, a high intensity beam is needed to collect a high luminosity to compensate the small cross-section of the Drell-Yan process. The absorber will be made of aluminum oxide $\left(\mathrm{Al}_{2} \mathrm{O}_{3}\right)$ with a tungsten core to stop the beam which does not interact in the target. The target platform will be moved upstream along the beam line to make enough room to place the hadron absorber, whose design is shown in Fig. 4. The absorber is made up of several aluminum oxide layer with increasing transverse dimension to cover the acceptance of the spectrometer. The layers are surrounded by stailess steel structures. An additional aluminium conical structure is in front of the layer and a removable stainless steel layer is placed at the end of the absorber. A tungsten beam plug is placed in the center of the absorber structure to stop the beam which did not interact with the target. The whole absorber is screened by concrete for radioprotection reason. Another upgrade is the vertex detector tracker which will be placed after the target, between the conical and the rectangular parts of the absorber. The vertex detector will help improving the spacial resolution of primary Drell-Yan vertexes and mass resolution of virtual photon.

Monte Carlo simulations and particle flux studies were done to get a design which fulfils physics requirements, as well as radioprotection rules and engineering needs. Simulations allowed to study 


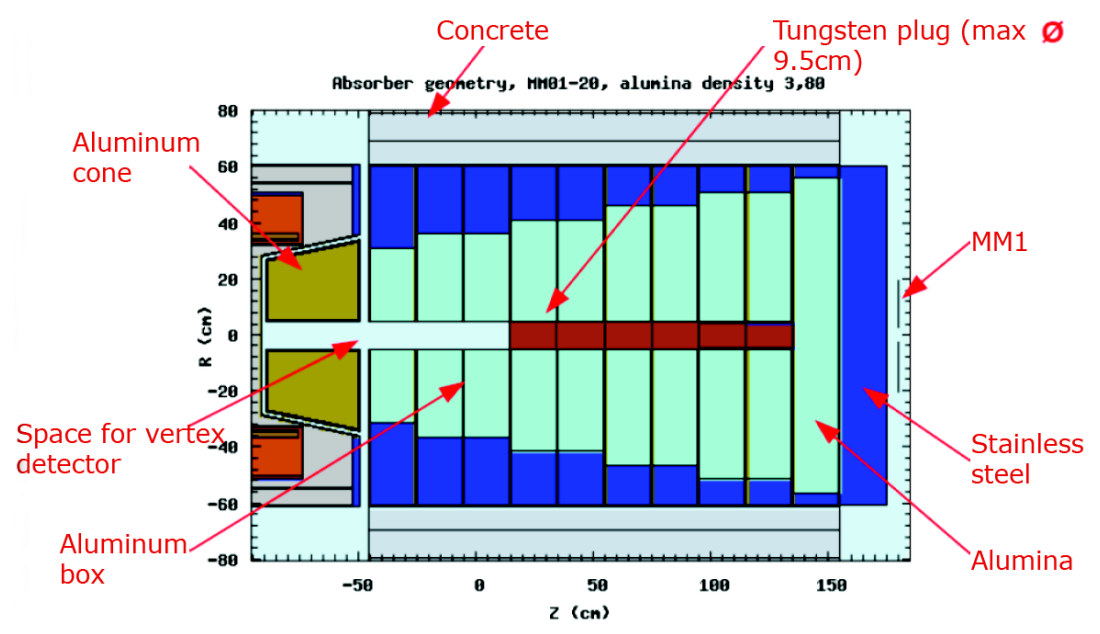

Figure 3: Detailed structure of the hadron absorber. It is made of ten $\mathrm{Al}_{2} \mathrm{O}_{3}$ layers surrounded by stainless stell and concrete. An additional aluminium conical structure is placed in the front of it. A removable stainless steel layer is hang at the end of the absorber.

the acceptance of the COMPASS spectrometer to Drell-Yan events. The study was done looking at the mass region of the virtual photon between 4 and $9 \mathrm{GeV} / \mathrm{c}^{2}$, the so-called "safe region" which is almost background free compared to the region below $3.5 \mathrm{GeV} / \mathrm{c}^{2}$.

\section{Beam tests}

In the last few years, short Drell-Yan beam tests were performed at COMPASS. At the end of 2009, an important test was done in conditions close to the future Drell-Yan measurement with a hadron absorber. Several results were achieved: the feasibility of the measurement was proven, the yields of $\mathrm{J} / \psi$ and Drell-Yan events were measured and the possibility to separate the Drell-Yan events coming from two different target cells was shown. During the test, radioprotection limits were respected, the detector occupancies were low and a good agreement was found between Monte Carlo simulations and reality.

\section{Predictions}

Single spin asymmetries are expected to be quite large in the proton valence region, which is covered by the COMPASS acceptance to Drell-Yan events. Several predictions are available for the COMPASS kinematic and 4-9 GeV/c ${ }^{2}$ virtual photon mass range: The projections of the statistical error on the Sivers asymmetry as compared to the various theoretical predictions, corresponding to two years of data taking, are shown in Fig. 6. These predictions do not take into account TMD evolution.

\section{Conclusion}

The COMPASS experiment has the possibility to give new results in the understanding of 


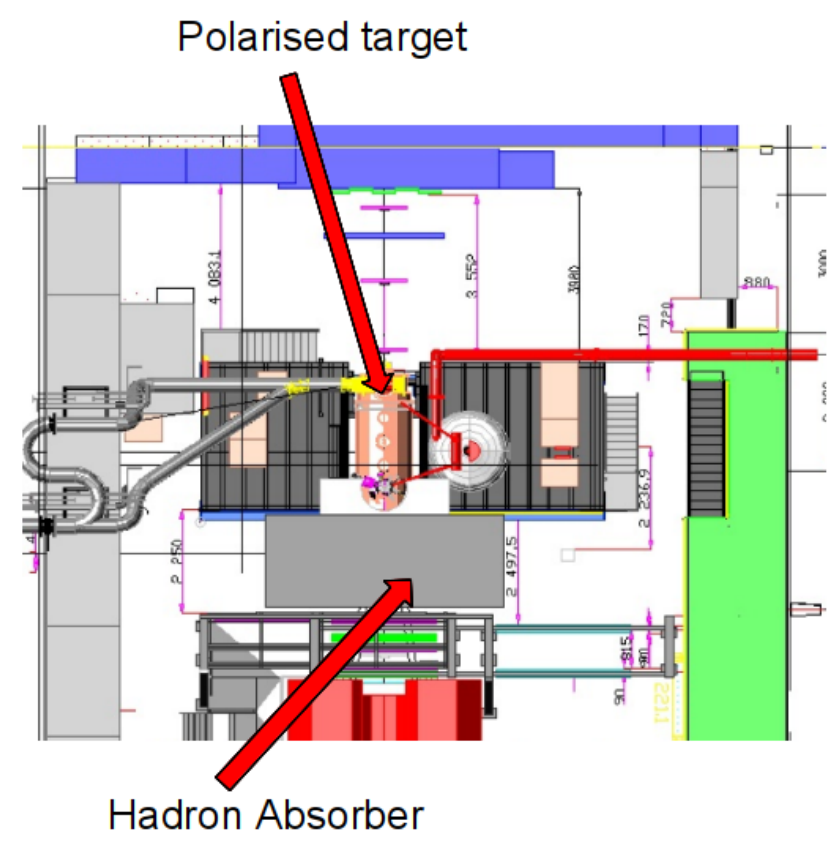

Figure 4: Top view of the target area of the COMPASS spectrometer. Beam comes from up.

the physics of the spin of the proton using single polarised Drell-Yan process, probing the TMD PDFs. An important test of the TMD factorization approach in QCD can be performed by checking whether the fondamental QCD prediction $\left(f_{1 T}^{\perp}(D Y)=-f_{1 T}^{\perp}(S I D I S)\right.$ and $\left.h_{1}^{\perp}(D Y)=-h_{1}^{\perp}(S I D I S)\right)$ is correct. The preliminary schedule of the COMPASS-II program foresees the start of the DrellYan measurent in late 2014.

\section{References}

[1] F. Gautheron et al., COMPASS-II Proposal, SPSC-P-340 (2010)

[2] J. C. Collins, Leading-twist Single-transverse-spin asymmetries: Drell-Yan and Deep-Inelastic Scattering, Phys. Lett. B536 (2002) 43-48 [hep-ph/0204004]

[3] S. D. Drell and T.-M. Yan, Massive Lepton Pair Production in Hadron-Hadron Collisions at High-Energies, Phys. Rev. Lett. 25 (1970) 316-320

[4] C. S. Lam and W.-K. Tung, A systematic approach to inclusive lepton pair production in hadronic collisions, Phys. Rev. D18 (1978) 2447

[5] S. Falciano et al., Angular distributions of muon pairs produced by $194 \mathrm{GeV} / \mathrm{c}$ negative pions, $\mathrm{Z}$. Phys. C31 (1986) 513

[6] J. S. Conway et al., Experimental Study of Muon Pairs Produced by 252-GeV Pions on Tungsten, Phys. Rev. D39 (1989) 92-112

[7] S. Arnold and A. Metz and M. Schlegel, Dilepton production from polarized hadron hadron collisions, Phys. Rev. D79 (2009) 034005 


\section{$\mathbf{A}_{\mathrm{T}}$}

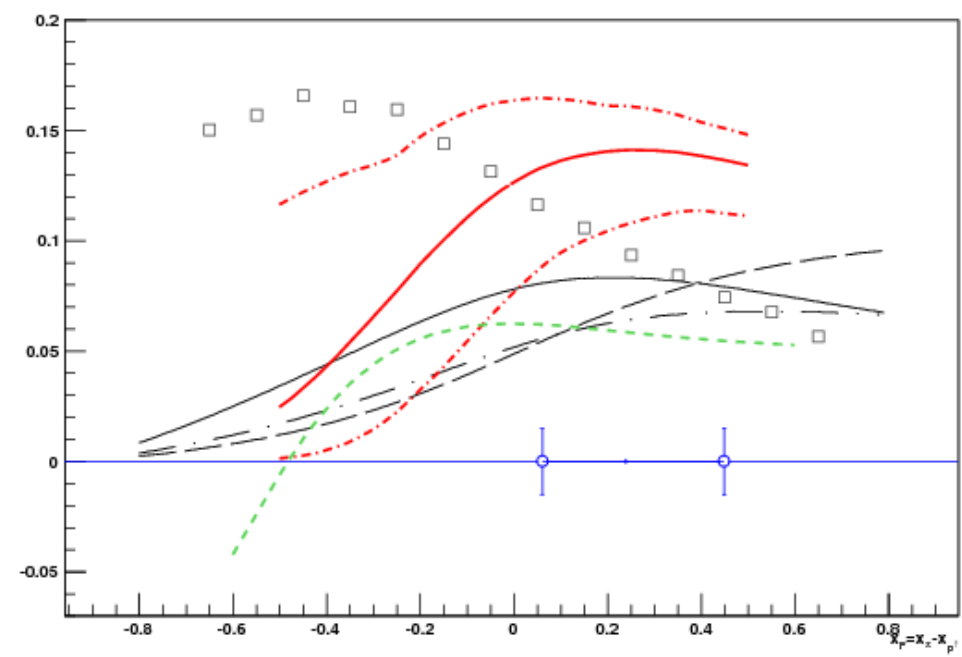

Figure 5: Comparisons of several prediction of the asymmetry term $A_{T}^{\sin \phi_{S}}$ in the COMPASS kinematic range and 4-9 GeV/c ${ }^{2}$ virtual photon mass range: black solid and dashed [11], dot-dashed [12], red [13], boxes [14], green short dashed [15]. In blue points with error bars coming from a two bin analysis and two years of data taking.

[8] J. C. Collins and D. E. Soper, Angular Distribution of Dileptons in High-Energy Hadron Collisions, Phys. Rev. D16 (1977) 2219

[9] G. Baum et al., COMPASS: A Proposal for a Common Muon and Proton Apparatus for Structure and Spectroscopy, CERN-SPSLC-96-14 (1996)

[10] P. Abbon et al., The COMPASS experiment at CERN, Nucl. Instrum. Meth. A577 (2007) 455-518 [hep-ex/0703049]

[11] A. V. Efremov, K. Goeke, S. Menzel, A. Metz, and P. Schweitzer, Sivers effect in semi-inclusive DIS and in the Drell-Yan process, Phys. Lett. B612 (2005) 233-244 [hep-ph/0412353]

[12] J. C. Collins et al., Sivers effect in semi-inclusive deeply inelastic scattering, Phys. Rev. D73 (2006) 014021 [hep-ph/0509076]

[13] M. Anselmino et al., Sivers effect in Drell-Yan processes, Phys. Rev. D79 (2009) 054010

[14] A. Bianconi and M. Radici, Monte Carlo simulation of single spin asymmetries in pion proton collisions, Phys. Rev. D73 (2006) 114002 [hep-ph/0602103]

[15] A. Bacchetta, F. Conti and M. Radici, Transverse-momentum distributions in a diquark spectator model, Phys. Rev.D78 (2008) 074010 